\title{
Activité professionnelle indépendante ou salariée des médecins en milieu hospitalier*
}

\author{
T. Eichenberger
}

\section{Principes}

La distinction entre revenus provenant d'une activité indépendante et revenus provenant d'une activité salariée et, partant, la qualification d'un assuré pour une activité déterminée, revêt une grande importance dans l'AVS (loi fédérale du 20 décembre 1946 sur l'assurance-vieillesse et survivants; RS 831.10) parce que la perception de la cotisation et les taux appliqués sont différents selon la situation.

Pour les salariés, la cotisation est perçue à la source, c'est-à-dire chez l'employeur, tandis que les indépendants doivent payer eux-mêmes la cotisation dans son entier. Aujourd'hui, la portée de cette distinction ne se limite pas à l'AVS/AI/PC. Elle exerce aussi ses effets sur la classification des assurés dans l'AC (assurancechômage), l'AA (assurance-accidents) et la PP (prévoyance professionnelle).

Concernant le statut juridique du médecin dirigeant, il apparaît en outre que les revenus d'une activité indépendante, obtenus sur la base d'une autorisation d'exercer à titre accessoire, ne sont pas limités vers le haut alors que les salaires le sont systématiquement, même quand il s'agit de revenus complémentaires.

Les possibilités juridiques octroyées à l'administration hospitalière d'influer sur l'activité du médecin dirigeant augmentent fortement lorsque seules des activités salariées sont encore exercées, c'est-à-dire dans le cadre d'un contrat d'engagement. Et le fait que l'employeur ne puisse guère imposer de directives concernant les soins donnés par le médecin dirigeant à son patient ne change rien à cette situation.

On notera enfin que l'autorité fiscale traite le salaire complémentaire tout autrement que l'activité indépendante exercée à titre accessoire.

4 mars 2003 à Zurich à l'occasion du séminaire de formation postgraduée de l'Université de St-Gall sur des problèmes actuels de droit dans le domaine médical.

Correspondance:

Dr en droit Thomas Eichenberger

Secrétaire général de l'AMPHS

Kellerhals \& Partner

Kapellenstrasse 14

CH-3011 Berne

\section{Les honoraires provenant d'une activité privée dans le domaine stationnaire, qui peuvent être facturés essentiellement à des patients couverts par une assurance complémentaire selon la LCA \\ Activité médicale privée dans le domaine stationnaire}

(loi sur le contrat d'assurance) et avec lesquels le médecin d'hôpital agréé conclut un contrat spécial de traitement, ne peuvent être déclarés à l'autorité fiscale comme revenus provenant d'une activité indépendante qu'à des conditions extrêmement restrictives en ce qui concerne le caractère indépendant de cette activité. De ce fait, et à bon droit, la plupart des caisses cantonales de compensation AVS ont commencé à considérer cette activité comme relevant d'un contrat de travail de droit public.

La perception de cotisations sur le revenu provenant d'une activité privée dans le domaine stationnaire est sans importance pour la délimitation juridique opérée par l'AVS. Si des cotisations sont perçues, ce fait ne prouve pas le caractère salarié ou indépendant de l'activité, selon le Tribunal fédéral. Cette perception peut aussi être une limitation du salaire complémentaire correspondant. Aucune attention n'est accordée aux conventions contractuelles ne concordant pas avec la législation AVS et la jurisprudence. Dans le jugement prononcé par le tribunal de Lucerne, le Tribunal fédéral des assurances (TFA) constate clairement et sans équivoque possible que la définition figurant dans l'ordonnance cantonale concernant les médecins-chefs, selon laquelle les honoraires provenant d'une activité privée dans le domaine stationnaire sont des revenus LPP produits par une activité indépendante et que l'affiliation à une institution de prévoyance pour ces revenus est l'affaire des médecins concernés, ne change rien à la classification prévue par l'AVS.

Dans ce contexte, il est important de mentionner d'emblée que les revenus considérés jusqu'à présent comme provenant d'une activité indépendante et constituant désormais un salaire déterminant pour l'AVS, doivent être assurés selon la LPP (loi fédérale du 25 juin 1982 sur la prévoyance professionnelle vieillesse, survivants et invalidité; RS 831.40) pour la partie obligatoire ou peuvent l'être pour le domaine dépassant la partie obligatoire. Les parts de revenus constituant le salaire déterminant pour l'AVS ne peuvent, dans le domaine situé au-dessus de la partie obligatoire, être assurées selon la LPP que par l'institution de prévoyance de l'employeur, si ses statuts le prévoient. 
Si cette possibilité n'existe pas, la Fondation pour la prévoyance professionnelle des médecins dirigeants d'hôpitaux de Suisse est la seule à offrir un plan de prévoyance sous forme d'assurance spéciale pour cadres. Pour les revenus élevés provenant d'une activité salariée, les médecins peuvent économiser et assurer jusqu'à 20\% desdits revenus à des conditions fiscalement avantageuses. La seule condition à remplir par les médecins en activité dans l'hôpital concerné est d'être membres de l'organisation professionnelle des médecins dirigeants d'hôpital ou de l'Association des médecins principaux d'hôpitaux de Suisse (AMPHS) dont le siège est à Berne.

Dans ce contexte, je me permets de vous renvoyer à une communication du professeur W. Schweizer, président de la Fondation pour la prévoyance professionnelle de l'AMPHS, qui sera prochainement publiée dans le Bulletin des médecins suisses.

\section{Consultation privée}

Selon la jurisprudence constante du TFA, les revenus issus de consultations privées à l'hôpital constituent en revanche, depuis toujours, des revenus provenant d'une activité indépendante. Les revenus provenant de consultations privées peuvent être assurés dans le cadre du $2^{\mathrm{e}}$ pilier facultatif (prévoyance professionnelle selon la LPP) auprès de la fondation de prévoyance de l'AMPHS et ils sont fiscalement déductibles.

Consultation privée veut dire séparation géographique, organisationnelle et personnelle avec l'entreprise hospitalière et donc aussi mise sur pied d'égalité juridique avec un cabinet médical à l'extérieur de l'hôpital. La jurisprudence constante du TFA précise que les honoraires facturés par des médecins-chefs pour le traitement et les soins de patients ambulatoires dans un local de consultation privé, mis à disposition par l'hôpital, sont des revenus provenant d'une activité indépendante. Elle ne s'applique néanmoins qu'à ces cas précis.

Lorsque cet état de faits n'est pas donné, en particulier quand l'hôpital prodigue lui-même des soins ambulatoires, l'aspect indépendant de l'activité des médecins-cadres n'existe plus.

Ici, il est déterminant de savoir qui, en sa qualité de fournisseur de soins, conclut le contrat de traitement avec le patient. Si les conditionscadres correspondantes sont remplies, la consultation privée à l'hôpital est assimilable à une consultation en cabinet à l'extérieur de l'hôpital.

Le fait que l'investissement nécessaire pour un cabinet médical destiné aux consultations privées soit moins élevé et que l'activité soit soumise à certaines restrictions dues aux conditions de travail, du moins en ce qui concerne les heures de consultation, ne joue aucun rôle. Le médecincadre ne se consacre pas pour autant, dans le cadre de ses consultations privées, à une activité typiquement dépendante du point de vue de l'organisation du travail.

La classification AVS ne peut pas donner lieu à des généralités. Les deux exceptions au sujet desquelles le TFA s'est prononcé dans le cadre de la jurisprudence actuelle, concernent d'une part un médecin-chef en radiologie dont l'activité devait être considérée comme des traitements ambulatoires à l'hôpital. Dans ce cadre, l'hôpital s'est présenté comme le fournisseur de prestations. Le médecin-chef radiologue concerné ne disposait ni d'un local en propre ni d'un personnel privé. Et, d'autre part, un arrêt de la $2^{\mathrm{e}}$ chambre de droit public, concernant d'abord une problématique relevant du droit des cotisations et non pas le statut AVS.

Il n'est pas possible de suivre en tous points les conclusions du Tribunal fédéral en l'espèce pour le statut non-indépendant AVS des revenus provenant de l'activité ambulatoire à l'hôpital universitaire de Genève, car le Tribunal fédéral estime, entre autres motifs principaux, que les tarifs habituels sont applicables pour les patients ambulatoires, ce qui - par analogie - n'entrerait pas dans le cadre d'une activité privée. En raison de la protection tarifaire existante selon l'art. 44 LAMal (loi fédérale du 18 mars 1994 sur l'assurance-maladie; RS 832.10), le tarif applicable n'est justement pas un critère d'appréciation utilisable.

Néanmoins, il ressort de l'arrêt du TFA que les hôpitaux ont la possibilité de supprimer les consultations privées pour les transformer en activité hospitalière ambulatoire.

\section{Recommandations actuelles de la Conférence des directeurs sanitaires}

Etant donné les recommandations sur la rémunération future des médecins d'hôpitaux du 17 octobre/ 7 novembre 2002, élaborées par la commission «Application LAMal» de la Conférence des directeurs des affaires sanitaires (CDS), la distinction juridique entre l'activité générale des médecins d'hôpitaux et l'activité privée doit être abolie parce qu'elle est dépassée.

A l'avenir, les médecins-chefs devraient exercer toute leur activité uniquement dans le cadre d'un contrat d'engagement (y compris les consultations privées). Dans une lettre du 
7 novembre 2002 adressée au président de l'AMPHS, le secrétaire central de la CDS prétend même que le médecin ne peut faire valoir les honoraires provenant d'une activité privée dans le domaine ambulatoire (consultations privées) comme revenus provenant d'une activité indépendante qu'à des conditions extrêmement restrictives en ce qui concerne le caractère indépendant de ladite activité. La CDS s'appuie à cet effet sur la dernière jurisprudence en date.

La CDS est en contradiction avec les faits ressortissant de l'activité menée dans le cadre de consultations privées et avec la jurisprudence constante du TFA, en vigueur depuis des années.

Des exceptions sont certes envisageables mais elles ne changent rien au principe. La recommandation de la CDS n'est, ni plus ni moins, qu'un projet politique visant à supprimer les consultations privées.

A mon avis, les médecins-cadres s'opposent à bon droit à la suppression des consultations privées. Elles doivent être maintenues parce qu'elles permettent d'éclaircir des cas difficiles à des conditions avantageuses.

Si cette activité n'est plus exercée en tant que consultation privée du médecin-cadre mais comme prestation hospitalière ambulatoire, le premier médecin n'enverra plus aucun patient à l'hôpital, car il veut que le médecin-cadre examine personnellement ses patients ou parce que le traitement n'est plus donné personnellement par le médecin-cadre contrairement à sa demande, ce qui revient finalement au même.

\section{Jurisprudence du Tribunal fédéral et littérature}

\section{a) Jurisprudence concernant le domaine hospitalier}

- ATF 101 V 252 (Zürich, médecin-chef radiologue salarié, soins stationnaires); $122 \mathrm{~V} 281$ (Lucerne, médecin salarié, soins stationnaires); $124 \mathrm{~V} 97$ (Genève, médecin salarié, soins stationnaires); H 201/00 du 19 mars 2002 (Berne, médecin-chef radiologue salarié, soins stationnaires).

\section{b) Jurisprudence concernant les consultations} privées (domaine ambulatoire)

- Principe: ATF 101 V 252, E. $1 \mathrm{~b}$ avec renvoi à ATFA 1967 p. 80 ss. ; cf. à la fin H 201/00 du 19 mars 2002, E. $3 a$, p. 6

- Exceptions: H 201/00 du 19 mars 2002 (Berne, médecin-chef radiologue salarié, traitements ambulatoires et dans le cadre de consultations privées) et Pra 1/1999, $\mathrm{n}^{\circ} 3$, p. 11 ss. (Genève, médecin salarié, traitements ambulatoires et dans le cadre de consultations privées). L'arrêt cité en dernier de la $2^{\mathrm{e}}$ Chambre de droit public du 11 août 1998 (Pra $1 / 1999, n^{\circ} 3$, p. 11 ss.) se réfère ainsi aux consultations pour des traitements ambulatoires mais pas les arrêts suivants du TFA cités à la p. 8 des recommandations de la CDS: ATF 122 V 281 (Lucerne, médecin salarié, soins stationnaires) et ATF $124 \mathrm{~V} 97$ (Genève, médecin salarié, soins stationnaires).

c) Importance de la taxation sur les revenus provenant d'une activité privée

- Cf. Pra 1/1999, n³ 3, E. 3cc, p. 20: il peut aussi s'agir de la limitation d'un salaire complémentaire; l'opinion défendue dans le cadre de ma dissertation, selon laquelle cela peut être considéré au sens large comme contribution des coûts de gain effectifs de l'activité privée, ne peut plus être maintenue à la lumière de la jurisprudence du TFA mentionnée.

\section{d) Bibliographie succincte}

- Käser H. Unterstellung und Beitragswesen in der obligatorischen AHV. 2. Auflage. Bern: Stämpfli; 1996.

- Eichenberger T. Die Rechtsstellung des Arztes am öffentlichen Spital. Dissertation. Bern/Stuttgart/ Wien; 1995.

- Wegleitung über den massgebenden Lohn (WML) des Bundesamts für Sozialversicherung vom 1. Januar 2002.

- Schaeppi C. Spitalarzt - Arbeitnehmer oder Selbständigerwerbender? Schweiz Ärztezeitung 1979; 60(11):505-8.

- Zuppinger F. Sozialversicherungsrecht und Steuerrecht, in: Sozialversicherungsrecht im Wandel. Festschrift 75 Jahre EVG. Bern: Stämpfli; 1992. S. 385-406. 